\title{
THE EFFECTIVENESS OF A MOBILE HEALTH APP FOR TAILORED HEALTHCARE EDUCATION WITH A MIGRANT INDIAN POPULATION IN HONG KONG
}

\author{
Matthew William Fendt ${ }^{1}$, Shelby Garner ${ }^{1}$, Phil Young ${ }^{1}$, Carolin George ${ }^{2}$, \\ Cho Lee Wong ${ }^{3}$, Hope Koch ${ }^{1}$, Gina Green ${ }^{1}$ and Julia Hitchcock ${ }^{1}$ \\ ${ }^{1}$ Baylor University, USA \\ ${ }^{2}$ Bangalore Baptist Hospital, India \\ ${ }^{3}$ The Chinese University of Hong Kong, Hong Kong
}

\begin{abstract}
Mobile health applications can allow for care providers to reach a broader audience than in-person interventions can. Additionally, in times of a pandemic like COVID-19, care can be provided in a safer way for providers and for patients. A mobile health app was developed to teach about the prevention and treatment of hypertension and diabetes. This app was specifically designed and tailored for an Indian population since these noncommunicable diseases are large contributors to death in Indian adults. Previous work targeted an Indian population in India, but this work explores the impact of the app with a migrant Indian working population in Hong Kong. Participants showed statistically significant learning gains in knowledge about both hypertension and diabetes as a result of using the app's animations and games. This demonstrates the effectiveness of mobile health apps at providing targeted healthcare education.
\end{abstract}

\section{KEYWORDS}

Mobile Health, Health Care, Learning Games

\section{INTRODUCTION}

The World Health Organization (WHO, 2019a) describes mobile health, or mHealth, as the use of mobile or wireless technologies to improve public health outcomes and advocates for its use among vulnerable populations who may have poor access to healthcare. The evolution of cell phones from 2-way analog devices to smartphones in recent years has provided an accessible pathway for interactions between health providers and public health populations (Garner et al., 2018; Sondaal et al., 2016). mHealth applications (apps) are increasingly designed to promote 
IADIS International Journal on WWW/Internet

a wide range of health interventions such as public health education, disease prevention, diagnosis and management (Haenssgen \& Ariana, 2017). However, mHealth apps containing culturally relevant information to address health priorities among some vulnerable populations remain limited (Garner et al., 2018; Garner et al., 2021; Sondaal et al., 2016).

Noncommunicable diseases (NCD) such as hypertension and diabetes are among the top contributors of death among adults ages 30-70 in India. Asian-Indians have one of the highest mortality rates from these diseases in the South East Asia region (WHO, 2019b). These poor health outcomes extend to Asian-Indians who have migrated to Hong Kong where the migrant worker population is proliferating and make up at least $10 \%$ of the domestic workforce (Agyemang et al., 2019; Liu et al, 2013 \& Lestari \& Li, 2019). In a clinical trial of 760 migrants from India to China, diabetes and hypertension outcomes (glycemic control and blood pressure) were poorer than that of Chinese nationals (Department of Family Medicine, Queen Elizabeth Hospital, 2014). Health providers recognize there is an urgent need for culturally relevant health resources for this population to improve health knowledge and health outcomes.

The coronavirus disease 2019 (COVID-19) pandemic and post-COVID-19 syndrome heightens the need for hypertension and diabetes prevention, management and treatment (Ayoubkhani et al., 2021; de Almeida-Pititto et al, 2020; Wu \& McGoogan, 2020). A summary article of 72,314 cases from the Chinese Center for Disease Control and Prevention reported cardiovascular disease and diabetes were the top two related preexisting conditions among people who died from COVID-19 (Wu \& McGoogan, 2020). Rates of cardiovascular disease $(126 / 1000$ cases $)$ and diabetes $(127 / 1000$ cases $)$ were found to be significantly raised $(\mathrm{p}<.001)$ among patients with COVID-19 in a retrospective cohort study of 47780 individuals aimed at quantifying rates of organ specific dysfunction in patients with COVID-19 after discharge from the hospital when compared with a control group (Ayoubkhani et al., 2021). Through a recent meta-analysis of 40 research articles involving 18,012 COVID-19 patients, researchers found diabetes and hypertension were moderately associated with COVID-19 related severity and mortality (de Almeida-Pititto et al., 2020). The COVID-19 pandemic expanded the need for virtual interventions such as mHealth educational resources to reduce COVID-19 transmission risk among patients and health providers. According to O'Connor and Bowles, virtual encounters among health providers and patients at one health system increased from 200 per month to 5000 a day in just 3 weeks. Mobile health apps are a valuable resource to aid health providers in continuity of care amidst lock down orders and high risk transmission periods.

We sought to create a mHealth app that teaches Asian Indian migrants to Hong Kong about hypertension and diabetes in an engaging and accessible way. A combined hypertension-diabetes app that contained culturally relevant videos and also small games and other engagements was developed for a specifically Indian population. We evaluated the app's effectiveness at teaching about hypertension and diabetes through a pre/post-test evaluation and found statistically significant improvements in participants regarding both hypertension and diabetes.

\section{RELATED WORK}

Mobile health initiatives have shown effectiveness in improving health outcomes, particularly in low and middle income countries (LMICs) (Sondaal et al., 2016). A systematic review was conducted to assess the impact of mHealth interventions on improving maternal and neonatal 


\section{THE EFFECTIVENESS OF A MOBILE HEALTH APP FOR TAILORED HEALTHCARE EDUCATION WITH A MIGRANT INDIAN POPULATION IN HONG KONG}

care in LMICs. Authors found mHealth interventions contributed to health service utilization including increased use of birth attendants and improved vaccination rates. However, few mHealth studies have focused on improving NCD outcomes among Indian populations. In a systematic review of clinical trials to assess the health-related impacts of mHealth on NCDs in LMICs, only 2 out of 8 studies were included from India (Stephani et al., 2016). These studies focused on the use of mHealth apps to improve compliance related to diabetes (Shetty et al., 2011) and cardiovascular disease (Tian et al., 2015). Results from the systematic review indicated mHealth interventions were primarily positive in promoting health, improving health appointment follow up compliance and providing clinical decision support systems (Stephani et al., 2016).

Our multidisciplinary and international mHealth team was composed of researchers and industry professionals from Computer Science, Nursing, Medicine, Management Information Systems, Art, Animation and Information Technology from the United States and India. The team collaborated from 2017-2020 to design and test two mHealth NCD apps among participants in Bengaluru, India. In 2018, our hypertension app was designed and tested among $\mathrm{N}=346$ participants living in rural villages (48.3\%) and urban slums $(51.7 \%)$ (Garner et al., 2021). A single arm pre/post-test design was used. Results indicated that the app was effective in improving hypertension health knowledge among participants ( $\mathrm{p}<.0001)$.

In 2020, a clinical trial was conducted to assess the effectiveness of our diabetes app in improving diabetes knowledge among $\mathrm{N}=291$ participants in an urban slum in India (Garner $\&$ Hitchcock, 2020). The intervention group who used the diabetes mHealth app $(\mathrm{N}=142)$ was compared with a control group $(\mathrm{N}=149)$ who received oral counseling on diabetes, the standard of care in India. While both groups yielded statistically significant improvements in knowledge after learning about diabetes $(\mathrm{P}<.0001)$ there was no statistically significant difference between post test scores between groups. Since the mHealth app was at least as effective as face to face oral counseling, the mHelath app showed promise as a useful tool for providing health education. When implementing this study, we noticed the app provided standard evidence based education while the oral counseling received by the control group often varied. We also noted the mHealth app reduced health provider fatigue during the long days of trialing the app (Garner \& Hitchcock, 2020).

\section{APP DESIGN}

This study used an app that was a combination of the two health apps previously developed by the team. The first app was a 3D animated video designed to teach about hypertension. This app was not interactive, but just consisted of the instructional video. The second app was a 3D animated video that also used games and other interactions in addition to the video. It consisted of four 2-minute videos, each with a game or interaction presented afterwards designed to reinforce the video's instructional material. This app was designed to teach about diabetes. For the combined version of the app in this study, a 10 question pre and post-test was added for each of hypertension and diabetes. The overall flow of the app is shown in Figure 1. 


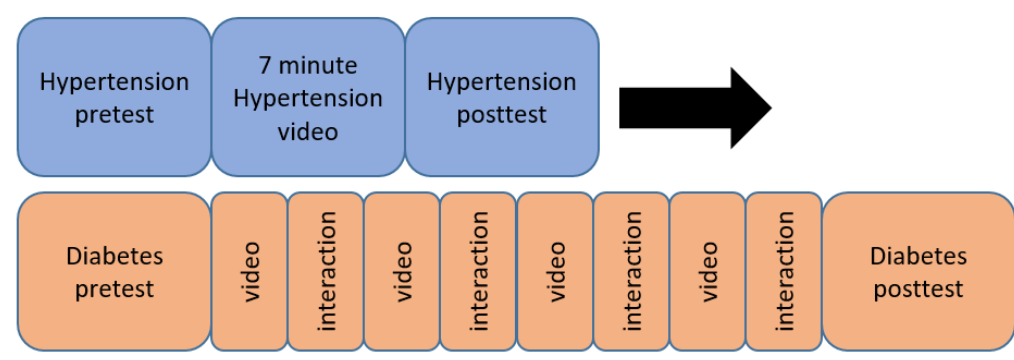

Figure 1. The structure of the combined mHealth app on hypertension and diabetes

Both apps were designed to teach about a particular disease that is especially prevalent to Indian culture. They were designed in collaboration with Indian doctors and software designers. Since the majority of the research team is American, these cultural resources were essential so we could target appropriate references to the audience. For example, we might have listed potato chips as an example of junk food, but our Indian team members suggested jalebi instead, which is a more common snack food in India. Another example of cultural advice our Indian team members provided was appropriate color and dress of the characters in our animation.

For the animation and interactions, we wanted to make sure to provide the content in an exciting and engaging manner. Another consideration is that although the material is serious, we wanted to make sure that it was not presented in a frightening fashion that would be a deterrent to the viewer and prevent them from engaging. An anthropomorphic animated monkey is the key character in both parts of the app. A narrator describes the dangers of the diseases and what can happen if it is left untreated, as well as how to prevent and treat the disease. The monkey character acts out both aliments and the treatments. The hypertension video takes place between three people as a casual interaction at a sales stand. In the diabetes video, guests at a wedding talk with a woman who has diabetes and is now successfully treating it. An example screenshot of the diabetes app is shown in Figure 2.

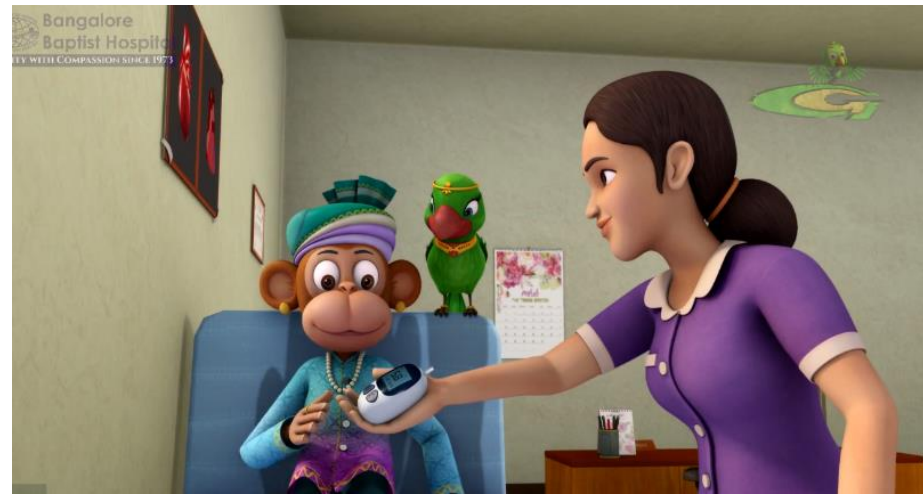

Figure 2. A screenshot of the instructional video portion of the app 


\section{THE EFFECTIVENESS OF A MOBILE HEALTH APP FOR TAILORED HEALTHCARE EDUCATION WITH A MIGRANT INDIAN POPULATION IN HONG KONG}

\section{GAMES AND INTERACTIONS IN THE APP}

The primary author was brought on the project at the beginning of creation of the second app, the one on diabetes. He helped develop the games and interactions that are interspersed in the diabetes app. The intention is to engage the viewer so that they are not just a passive observer. The viewer watches a two-minute video, then plays through an interaction that reinforces the section's instructional content. The interaction is designed to only take a few seconds to complete as to not break the immersion of the video.

The first small video is about how diabetes can be caused. The interaction reinforces the concept of insulin acting as a key to unlock the body's cells to be able to receive blood glucose. This is a simple interaction where the viewer sees an animation of the blood glucose unable to enter the cell. They then hit a button showing a key, and the video shows the insulin acting as a key to unlock the cells.

The second video discusses the dangers of untreated diabetes. The interaction presents the viewer with a series of animated buttons illustrating possible ailments caused by the disease. After they select each correct answer, the app reinforces their correct answer.

The third video shows how to eat in a healthy way to prevent and manage diabetes. In this interaction, the viewer is presented with a number of foods and a plate split into one half and two quarters. Following the MyPlate method of one-half fruits and vegetables and one-quarter grains and proteins, the viewer selects an appropriate meal. They must avoid junk food and choose correction portions for the food. This process is repeated two more times with different foods. This activity was the longest, most game-like, and involved of all four interactions. A screenshot of the game is shown in Figure 3.

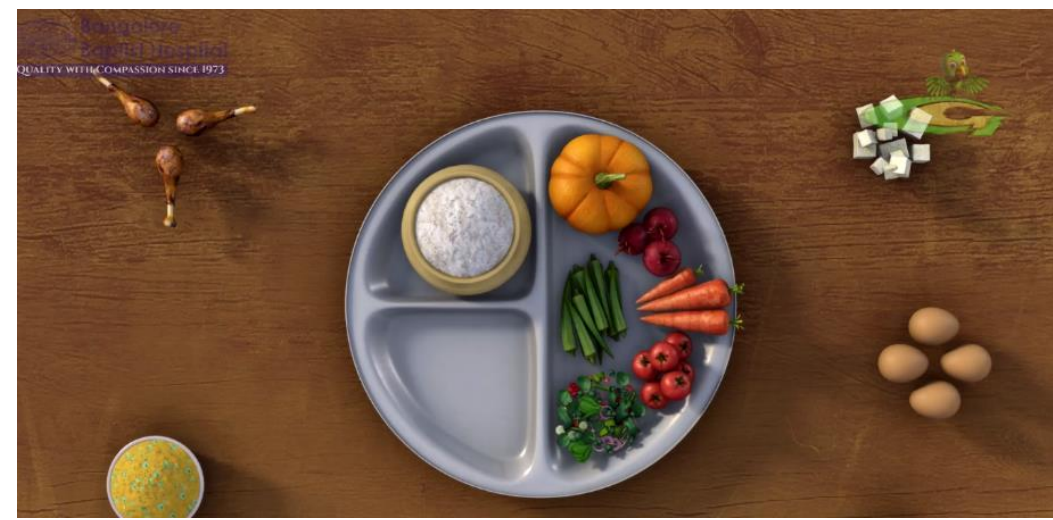

Figure 3. The plate game challenged participants to create a healthy meal and portion correctly in order to help prevent diabetes

Finally, the last video talks about healthy habits to prevent and manage diabetes. The interaction shows an animated loop of the happy monkey and his bird friend walking towards the user. Like the second interaction, the viewer is shown illustrated buttons with possible activities that one might do to combat diabetes, and after each correct selection, the viewer is given positive reinforcement. 
IADIS International Journal on WWW/Internet

The intention of all of these simple interactions is to engage the viewer so they are not completely passive. The interaction is meant to contribute to the learning aspect which is evaluated by the post-test.

\section{EXPERIMENTAL DESIGN}

Research Assistants fluent in English and Hindi recruited participants who were participating in activities associated with a variety of ethnic minority organizations in Hong Kong. 46 Indian people, living in Hong Kong, completed the study. They were asked a number of demographics questions, including how long they have lived in Hong Kong. 10 lived there less than one year, 19 lived there 1-10 years, and 17 lived there 11 years or more. Most (25) were in the 35-59 age group, with $18-34$ being the second most common. 28 were women, and 18 were men.

Among participants, $30.4 \%$ lived in urban Hong Kong and $59.6 \%$ were from rural areas. Most, $65.2 \%$, completed the app in English while 34.8\% completed it in Hindi. $8.7 \%$ had never attended school, while $91.3 \%$ had completed at least a $9^{\text {th }}-10^{\text {th }}$ grade education. The majority were employed at $73.9 \%$. $15.2 \%$ reported a history of diabetes or hypertension, 17.4 reported the use of cigarettes or smokeless tobacco and $41.3 \%$ reported alcohol use.

A researcher explained the purpose of the student and obtained consent from the participant. They completed the demographic information survey and then went through the pretest/ instruction/post-test sequence for hypertension and then diabetes. The research also collected qualitative feedback from the participant.

Our hypothesis is as follows:

Hypothesis: Participants that engaged with the instructional videos and interactions would show a statistically significant improvement on their post-test scores compared to their pretest scores.

\section{EXPERIMENTAL RESULTS}

A paired t-test was run on the pretest and post-test scores for both hypertension and diabetes. In both cases, there was a statistically significant improvement on the participants' post-test scores.

For hypertension, $t=5.498$, degrees of freedom $=45$, and the $p$ value was $1.725 \mathrm{e}-06$. The descriptive statistics for the hypertension pretest were: mean 6.48, standard deviation 2.008, median 7 and for the post-test were: mean 7.74, standard deviation 1.357, median 8 .

For diabetes, $t=8.46$, degrees of freedom $=45$, and the $p$ value was $7.467 \mathrm{e}-11$. The descriptive statistics for the diabetes pretest were: mean 6.413, standard deviation 2.578 , median 7 and for the post-test were: mean 9.5, standard deviation 1.188, median 10. A summary of these statistics is given in Table 1 . 


\section{THE EFFECTIVENESS OF A MOBILE HEALTH APP FOR TAILORED HEALTHCARE EDUCATION WITH A MIGRANT INDIAN POPULATION IN HONG KONG}

Table 1. A summary of the post-test improvement for the hypertension and diabetes sections of the app. Participants showed a statistically significant improvement in both sections

\begin{tabular}{|l|l|l|l|l|}
\hline & Pretest & Posttest & Improvement & $p$ value \\
\hline Hypertension & 6.48 & 7.74 & 1.26 & $1.725 \mathrm{e}-06$ \\
\hline Diabetes & 6.41 & 9.50 & 3.10 & $7.467 \mathrm{e}-11$ \\
\hline
\end{tabular}

The number of participants that got each question correct versus incorrect for the pre and post-tests was also measured. This data is shown in Table 2. Most participants got almost all of the questions correct in the post-test except for questions 6, 8, and 9 in the hypertension test. These questions were "Does high BP always cause symptoms?", "Someone having a stroke might have which happen?" and "The best way to know if I have high BP is to pay attention to abnormal feelings is:" It seems like these questions were either confusing or not taught well in the app, and account for the larger improvement in the diabetes test scores.

Table 2. Pre and post-test correct answers for hypertension and diabetes

\begin{tabular}{|c|c|c|c|c|c|c|c|c|c|c|}
\hline & Q1 & Q2 & Q3 & Q4 & Q5 & Q6 & Q7 & Q8 & Q9 & Q10 \\
\hline H pre & 33 & 36 & 26 & 36 & 34 & 25 & 43 & 19 & 9 & 37 \\
\hline H post & 45 & 42 & 38 & 44 & 44 & 20 & 46 & 28 & 7 & 42 \\
\hline D pre & 21 & 32 & 34 & 15 & 22 & 19 & 33 & 40 & 38 & 41 \\
\hline D post & 46 & 45 & 44 & 43 & 45 & 44 & 44 & 43 & 41 & 42 \\
\hline
\end{tabular}

To illustrate one possible confusion, 25 people answered the question "Does high BP always cause symptoms?" correctly in the pretest, but only 20 people answered it correctly in the post-test. This shows that people were confused by the app and changed their correct belief to an incorrect belief. The correct answer is "no," but over the course of the app, we showed many ways in which hypertension symptoms can manifest. However, hypertension is also sometimes referred to as the "silent killer" since it does not always have symptoms. This leads us to believe that by us showing numerous symptoms people were lead to believe that it always causes visible issues.

ANOVA was run on the improvement of the post-test scores and the demographic variables for both hypertension and diabetes. There was a statistically significant difference between people that had attended school versus not people that had attended school on their post-test scores ( $p=0.04$ for hypertension and $p=0.006$ for diabetes). Figure 4 summarizes this data. Those that had not attended school showed a larger gain in knowledge in both hypertension and diabetes, compared to the more modest gain for those that had attended school. Both groups had statistically significant gains overall and ended at a similar post-test score. However, those that had not attended school had their knowledge start low and ended high, as opposed to those that had attended school whose knowledge started medium and ended high. 

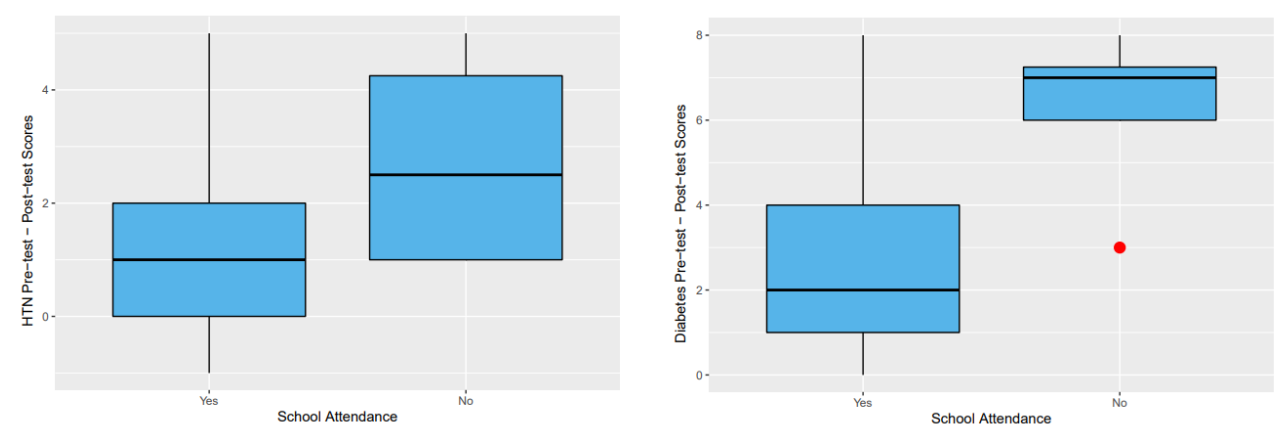

Figure 4: Those that have not attended school showed a statistically significantly larger improvement on their post-test scores, compared to those that have attended school, on both hypertension and diabetes

Additionally, we performed two canonical correlation analyses relating diabetes knowledge with hypertension knowledge first prior to viewing the app and also after viewing the app. In both analyses, four diabetes subscales comprised the set of independent variables and four hypertension subscales comprised the set of dependent variables. The subscale were sub-categories of knowledge about the disease. One subscale was omitted from both canonical correlation analyses due to the singularity of the correlation matrix. The R package "cca" yielded three canonical functions with their corresponding canonical correlations as presented in Tables 3 and 4 for pre-test and post-test knowledge, respectively.

Table 3. Canonical correlations for all considered canonical functions and the results of Bartlett's V test to assess significant significance of each canonical correlation

\begin{tabular}{|c|c|c|c|c|}
\hline $\begin{array}{c}\text { Canonical } \\
\text { function }\end{array}$ & $\begin{array}{c}\text { Canonical } \\
\text { correlation }\end{array}$ & $\begin{array}{c}\text { Squared Canonical } \\
\text { Correlation }\end{array}$ & $\begin{array}{c}\text { Bartlett's V } \\
\text { Chi-squared score }\end{array}$ & p-value \\
\hline 1 & 0.665 & 0.442 & 26.991 & $\mathbf{0 . 0 0 1 4}$ \\
\hline 2 & 0.233 & 0.054 & 2.756 & 0.5995 \\
\hline 3 & 0.103 & 0.011 & 0.439 & 0.5075 \\
\hline
\end{tabular}

Table 4. Measures of overall model fit for Diabetes and Hypertension post-test scores

\begin{tabular}{|c|c|c|c|c|}
\hline $\begin{array}{c}\text { Canonical } \\
\text { function }\end{array}$ & $\begin{array}{c}\text { Canonical } \\
\text { correlation }\end{array}$ & $\begin{array}{c}\text { Squared Canonical } \\
\text { Correlation }\end{array}$ & $\begin{array}{c}\text { Bartlett's V Chi-squared } \\
\text { score }\end{array}$ & p-value \\
\hline 1 & 0.694 & 0.482 & 28.984 & $\mathbf{0 . 0 0 0 7}$ \\
\hline 2 & 0.195 & 0.038 & 1.679 & 0.7945 \\
\hline 3 & 0.041 & 0.002 & 0.070 & 0.7920 \\
\hline
\end{tabular}

For our pre-test CCA model, three canonical functions were originally considered. Bartlett's $\mathrm{V}$ test for canonical functions, which uses the Chi-squared distribution, revealed that only the significance of the first canonical function exceeds the critical value at the 0.05 level. This result implies that both pre-test diabetes and hypertension comprehension and pre-test diabetes and hypertension comprehension will be conveyed in one dimension.

Table 5 shows the canonical loading values of the subscales for the diabetes and hypertension pretest and post-test. Like correlation, these loading values range on a scale of -1 to +1 . Adopting the criteria set in Baloglu et al. (1998) of canonical values of $>0.45$ or $<-0.45$ being significant, our analysis deemed relevant all three considered pre-test diabetes subscales but only one subscale for pre-test hypertension. 


\section{THE EFFECTIVENESS OF A MOBILE HEALTH APP FOR TAILORED HEALTHCARE}

EDUCATION WITH A MIGRANT INDIAN POPULATION IN HONG KONG

Table 5. Canonical loadings for diabetes and hypertension

\begin{tabular}{|c|c|c|}
\hline & $\begin{array}{c}\text { Canonical Loading } \\
\text { Pre test }\end{array}$ & $\begin{array}{c}\text { Canonical Loading } \\
\text { Post test }\end{array}$ \\
\hline Diabetes subscales & -0.6447 & 0.0910 \\
\hline $\begin{array}{c}\text { What can happen if I have } \\
\text { diabetes }\end{array}$ & -0.9501 & -0.8561 \\
\hline $\begin{array}{c}\text { How can I prevent and } \\
\text { manage diabetes }\end{array}$ & -0.7238 & -0.8407 \\
\hline What should I do now & -0.9692 & -0.9764 \\
\hline Hypertension subscales & -0.4097 & -0.2737 \\
\hline $\begin{array}{c}\text { What causes high blood } \\
\text { pressure }\end{array}$ & 0.0089 & -0.1807 \\
\hline $\begin{array}{c}\text { What happens if I have high } \\
\text { blood pressure }\end{array}$ & & \\
\hline What should I do now & & \\
\hline
\end{tabular}

The significance of having the subscales is that they show the different components of a person's knowledge on a subject. For example, according to the pretest, before using the app the participant's knowledge about diabetes consisted of a mix of all three areas: "what can happen if I have diabetes," "how can I prevent and manage diabetes," and "what should I do now." However, according to the pretest, before using the app the participants' knowledge about hypertension really only consisted of the "what causes high blood pressure category." This means that it would be important for the participant to learn about the other important components of hypertension by using the app.

Looking at the diabetes post-test canonical loading values, we see that the focus of participants' understanding has shifted from general knowledge to specific prevention and management strategies, as well as what they should do now. The absence of "what can happen if I have diabetes" does not indicate a loss of knowledge, but rather a refocusing on the other topic areas. This result is desirable and is likely due to the emphasis on the app being how to take action to fight the disease.

However, we fail to see much knowledge gain in hypertension topic areas other than "what causes high blood pressure." This doesn't mean that participants were not learning during the hypertension part of the app. Figures 1 and 2 do show learning gain, but primarily in the sub-category of "what causes high blood pressure." This is additionally problematic since they came without much knowledge of the other two sub-categories. These low loading values further emphasize the fact that hypertension questions 6,8 , and 9 need to be redesigned because they contributed to the failure of the participants learning the other two sub-categories.

Further analysis of Tables 3 and 4 shows the fact that one of the canonical functions is statistically significant for both pre and post-test which means that there is a relationship between the participant's knowledge and hypertension and diabetes before the app and also after the app. The correlation between hypertension and diabetes knowledge in the pretest was 0.665 , which indicates a moderately strong linear relationship for univariate latent variables of hypertension knowledge and diabetes knowledge for subjects prior to viewing the app. Therefore, participants' knowledge of one kind of disease was mildly predictive of their knowledge of the other kind of disease. This data is shown in Figure 5. 
IADIS International Journal on WWW/Internet

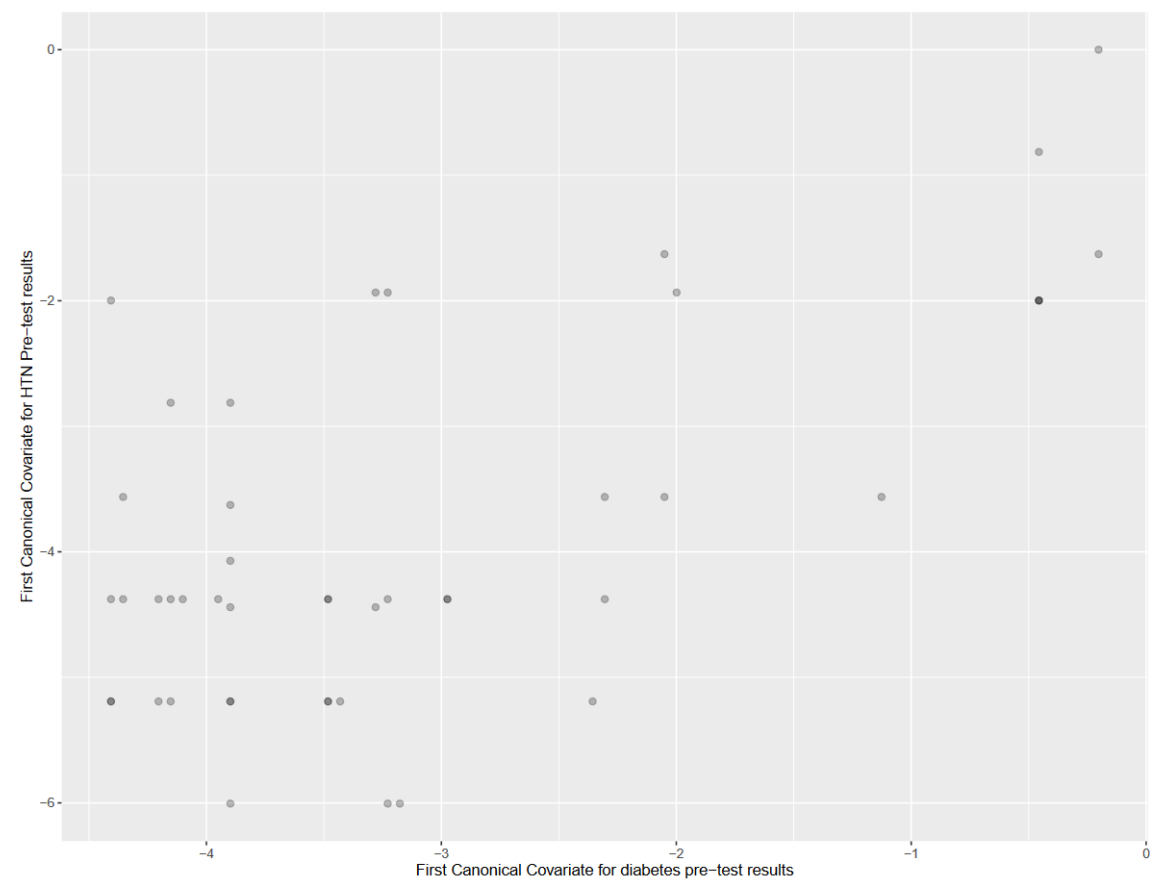

Figure 5. Scatterplot showing the relationship between pretest scores for diabetes and hypertension

Also, the correlation between hypertension and diabetes knowledge in the post-test was 0.694, which indicates a moderately strong linear relationship for the univariate latent variables of hypertension knowledge and diabetes knowledge for subjects after viewing the app. This data is shown in Figure 6. Again, knowledge after instruction about one disease was mildly predictive of knowledge after instruction about the other disease.

As a side note, while a linear trend between the first canonical variates is somewhat evident in the scatterplot, the cluster of observations in the bottom corner results from the fact that the vast majority of participants obtained a perfect score on the diabetes test after viewing the app. The darker dots in Figure 6 indicate that multiple data points are at the same location on the graph. 
THE EFFECTIVENESS OF A MOBILE HEALTH APP FOR TAILORED HEALTHCARE EDUCATION WITH A MIGRANT INDIAN POPULATION IN HONG KONG

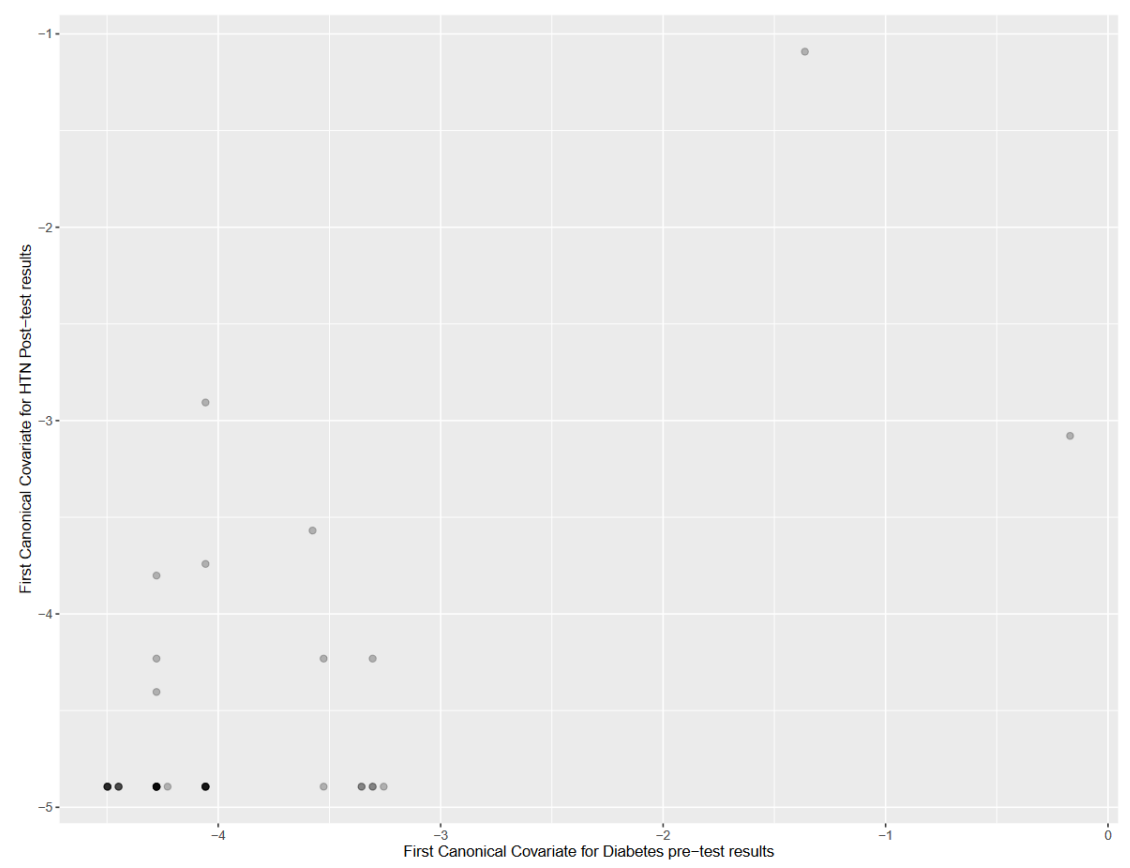

Figure 6. Scatterplot showing the relationship between pretest scores for diabetes and hypertension

\section{QUALITATIVE DATA}

Researchers conducted 18 after-study interviews to assess the participants' perception of the app's strengths and weaknesses. The perceptions addressed the app overall and the plate game.

Overall app and interaction: Several participants said they liked the interactive nature of the app, which has a cyclic learning process of showing and evaluating understanding of the material. They appreciated that the app was not scary, with one person saying: "even if somebody is suffering from something, it does not scare you." Five different people commented explicitly on the entertaining nature of the game, with one saying, "It's not a very boring doctor speech kind of a thing." Many people liked the monkey and the parrot mascots. We had some feedback that the app could be shown to a younger audience so that the intervention could begin at an early age or that the app could send out reminders on people's phones to reinforce the learning content.

Plate game specifically: Many people reported that they liked the plate game, citing that it was a useful tool at teaching healthy eating and proportions. One person said that "It's not only fun; you also learn. I didn't know that - I always thought you had to consume more protein than vegetables, but now I know through the app that I should consume more vegetables and one-fourth of protein, and one-fourth of carbohydrates." Some people suggested having more or different food options to choose from when constructing their plates or having fewer iterations of the game's plate composition aspect or having fewer iterations of the game's plate composition aspect. 


\section{DISCUSSION AND FUTURE WORK}

The app produced statistically significant learning gains in healthcare education about both hypertension and diabetes, demonstrating its effectiveness as a learning tool among an Asian Indian migrant population in Hong Kong. The app seemed disproportionately effective for those that have not attended school, emphasizing its impact among a vulnerable population with low health literacy. Also, people found the app interesting and engaging and participants found the plate game useful at learning healthy meal composition and proportions. It could be useful in the future to evaluate long-term learning that was a result of the app as opposed to just shortterm learning gains, or evaluate to see if learning from the app led to any change of behavior. Since the app was designed specifically for an Indian population, it is likely that a different population would not benefit from it as much as did the Indian population. However, a different population could also be evaluated to see how much of the learning gains would transfer over. If there is still sufficient learning gain, opening the app up to a general audience would allow for more people to benefit from it.

In addition to the app being an effective learning tool, creating and distributing an mHealth app can be easier than providing traditional medical interventions. In the traditional Indian model (Balagopal et al., 2008), healthcare workers go out to the people and teach about health care individually to the population. This direct intervention requires time, training, and expense. In contrast, delivering medical education with an app only requires publish the app in an available location and publicizing it to the target audience. Easily accessible electronic health education resources are particularly needed during the COVID-19 pandemic when face to face education could increase COVID-19 transmission, disease severity and death rates for high risk patients with hypertension and diabetes. Delivering health education via an app will also help protect health workers from acquiring COVID-19 as will reduce face to face contact. A future study could evaluate to see if there is a difference for the participants in learning between this app and a healthcare worker. To increase its accessibility, a modified version of this app has been published for free download on the Google Play store as "I Feel Great India." The prevalence of mobile devices greatly increases the accessibility of healthcare education.

\section{CONCLUSION}

A mobile health app was designed to teach about hypertension and diabetes, specifically targeting an Indian population. This app was evaluated for effectiveness in healthcare education among a migrant Indian population in Hong Kong. The research found statistically significant improvement in post-test scores about both hypertension and diabetes after interacting with the videos and games in the app. The app's efficacy, combined with the reach that mobile apps provide, shows a promising route for providing targeted healthcare education, especially to vulnerable populations. 


\section{THE EFFECTIVENESS OF A MOBILE HEALTH APP FOR TAILORED HEALTHCARE EDUCATION WITH A MIGRANT INDIAN POPULATION IN HONG KONG}

\section{REFERENCES}

Agyemang C, van den Born B-J, 2019. Noncommunicable diseases in migrants: an expert review. J Travel Med. 26(2). doi:10.1093/jtm/tay107

Ayoubkhani D. et al., 2020. Post-covid syndrome in individuals admitted to hospital with covid-19: retrospective cohort study. The bmj. 372(693), 1-10. doi:10.1136/bmj.n693

Balagopal P. et al, 2008. A community-based diabetes prevention and management education program in a rural village in India.By: Diabetes Care, 31(6), 1935-5548

Baloglu, S., Weaver, P., and Mccleary, K. W. (1998), Overlapping product-benefit segments in the lodging

industry: a canonical correlation approach, International Journal of Contemporary Hospitality Management, 10(4), pp. 159-166.

De Almeida-Pititto B. et al., 2020. Severity and mortality of COVID 10 in patients with diabetes, hypertension and cardiovascular disease: a meta-analysis. Diabetology \& Metabolic Syndrome, 12(75), 1-12. doi:10.1186/s13098-020-00586-4

Garner, S. et al, 2021. Effectiveness of an mHealth application to improve hypertension health literacy in India. International Nursing Review. 67(4), 476-483. doi:10.1111/INR.12616

Garner, S.L. et al, 2018. Smart phone accessibility and mHealth use in a limited resource setting International Journal of Nursing Practice, 24, e12609. https://doi.org/10.1111/ijn.12609

Garner S.L., Hitchcock J., 2020. Design and testing of mobile health applications to improve non-communicable disease health literacy among vulnerable populations in India. Baylor College of Medicine: Center of Excellence Health Equity Training and Research. 2020 Health Equity Summer Research Summit.

Haenssgen, M. J., \& Ariana, P, 2017. The social implications of technology diffusion: Uncovering the unintended consequences of people's health-related mobile phone use in rural India and China. World Development, 94, 286-304. http://dx.doi.org/10.1016/j.worlddev.2017.01.014

Lestari E, Li P, 2019. Fighting for Migrant Workers in Hong Kong. Mon Rev. Published online February 1, 2019 56-63. doi:10.14452/MR-070-09-2019-02 4

Liu S. et al, 2013. Comparison of Health Status and Health Care Services Utilization between Migrants and Natives of the Same Ethnic Origin-The Case of Hong Kong. Int J Environ Res Public Health. 10(2):606-622. doi:10.3390/ijerph10020606

O'Conner M., Bowles K. (2021). Telehealth and mHealth, Res Nurs Health. 44, 3-4. doi:10.1002/nur.22101

Shetty AS. et al, 2011. Reinforcement of adherence to prescription recommendations in Asian Indian diabetes patients using short message service (SMS)-a pilot study. J Assoc Physicians India. 59(7), $11-4$.

Sondaal, S. F. et al, 2016. Assessing the effect of mHealth interventions in improving maternal and neonatal care in low and middle income countries: a systematic review. PLoS One, 1-26. https://doi.org/10.1371/journal.pone.0154664

Stephani, V. et al, 2016. A systematic review of randomized controlled trials of mHealth interventions against noncommunicable diseases in developing countries. BMC Public Health, 16, 572. doi:10.1186/s12889-016-3226-3

Tian M. et al., 2015. A cluster-randomized controlled trial of a simplified multifaceted management program for individuals at high cardiovascular risk (SimCard Trial) in Rural Tibet, China, and Haryana, India. Circulation. 132(9):815-24.

World Health Organization, 2019a. WHO guideline: Recommendations on Digital Interventions for Health System Strengthening. https://www.who.int/publications-detail/who-guideline-recommendationsondigital-interventions-for-health-system-strengthening accessed 1 June 2019 . 
IADIS International Journal on WWW/Internet

World Health Organization, 2019b. Monitoring Health for the SDGs Sustainable Development Goals. https://www.who.int/publications/i/item/world-health-statistics-2019-monitoring-health-for-the-sdgssustainable-development-goals Accessed 1 December 2020.

Wu, Z. McGoogan, M., 2020. Characteristics of important lessons from the coronavirus disease 2019 (COVID-19) outbreak in China: Summary of a report of 72314 cases from the Chinese Center for Disease Control and Prevention. JAMA. 323(13). doi:10.1001/jama.2020.2648

Department of Family Medicine and GOPC, Queen Elizabeth Hospital, 30 Gascoigne Road, Kowloon, Hong Kong, Chen CX, Chan K. Type 2 diabetes management in Hong Kong ethnic minorities: what primary care physicians need to know. Hong Kong Med J. Published online January 30, 2014. doi:10.12809/hkmj134035 